\title{
FASTER HEALTH DETERIORATION AMONG NAIL TECHNICIANS OCCUPATIONALLY EXPOSED TO LOW LEVELS OF VOLATILE ORGANIC COMPOUNDS
}

\section{PETER GREŠNER, RADOSŁAW ŚWIERCZ, WOJCIECH WĄSOWICZ, and JOLANTA GROMADZIŃSKA}

\author{
Nofer Institute of Occupational Medicine, Łódź, Poland \\ Department of Toxicology and Carcinogenesis
}

\begin{abstract}
Objectives: The study has aimed at investigating the subjective assessment of an individual's health status and comparing the prevalence of selected work-related symptoms among nail technicians occupationally exposed to volatile organic compounds (VOCs) to the one among control subjects. Associations between occupational exposure to VOCs and the incidence of adverse health effects were also analyzed. Material and Methods: The study involved 145 female nail technicians and 152 control subjects. Data on the prevalence of adverse health effects was collected using the researcher-made questionnaire and then analyzed by means of survival analysis methods. Results: Only $22 \%$ of nail technicians as compared to $45 \%$ of control subjects described their current health status as "excellent" or "very good" (odds ratio $(\mathrm{OR})=0.4,95 \%$ confidence interval $(\mathrm{CI}): 0.2-0.6, \mathrm{p}<0.00005)$. In general, $61 \%$ of nail technicians confirmed to have experienced any out of all symptoms considered in the study since the commencement of the job, which was significantly higher as compared to $17 \%$ of control subjects (adjusted OR $=2.8,95 \%$ CI: 2.1-3.7, p $<0.0001$ ). Estimated median length of the employment period free of investigated symptoms was significantly shorter among nail technicians as compared to controls (12 years vs. 33 years, $p<0.0001$ ), consistent with almost 4-times increased hazard of the occurrence of such symptoms among the technicians (hazard ratio $(\mathrm{HR})=3.9,95 \% \mathrm{CI}$ : 2.7-5.7, p < 0.0001). Cox proportional hazard regression modeling revealed almost 5-times increased hazard of the occurrence of any symptoms among nail technicians exposed to higher levels of the mixture of VOCs as compared to those exposed to lower levels (HR $=4.9,95 \%$ CI: $1-24.1$, $\mathrm{p}=0.05)$. Conclusions: All outcomes combined together indicate that nail technicians are subject to faster health deterioration, which may be assumed to be caused by occupational exposure to low levels of VOCs. Int J Occup Med Environ Health 2017;30(3):469-483
\end{abstract}

Key words:

Occupational exposure, Volatile organic compounds, Survival analysis, Case-control study, Adverse health effects, Nail technicians

\footnotetext{
Funding: the study was supported by the National Science Centre grant "Effect of genetic variability on oxidative stress biomarkers and DNA damage caused by occupational exposure in nail technicians" No. PB/2011/01/B/NZ7/04681 (grant manager: Peter Grešner, Ph.D.) and the Nofer Institute of Occupational Medicine fund "Effect of polymorphisms of DNA repair genes on level of DNA damage caused by occupational exposure in nail technicians" No. IMP 1.18/2013 (fund manager: Peter Grešner, Ph.D.).

Received: November 18, 2015. Accepted: April 26, 2016.

Corresponding author: P. Grešner, Nofer Institute of Occupational Medicine, Department of Toxicology and Carcinogenesis, św. Teresy 8, 91-348 Łódź, Poland (e-mail: pgresner@imp.lodz.pl).
} 


\section{INTRODUCTION}

Nail technicians present an occupational group, the number of which has increased dramatically since the couple of last decades in all western countries [1]. According to the Central Statistical Office, it is estimated that more than 100000 registered nail technicians work in Poland (unpublished data). Services provided by nail technicians are unique in several specific aspects including contact with toxic and potentially dangerous chemical substances (such as ethanol, acetone, toluene, a number of acetates, acrylates, etc.), work in small and often poorly ventilated rooms as well as working procedures necessitating the use of chemical products in close vicinity to technician's breathing zone and eyes. Due to the fact that the concentrations of chemical products used in this industry are very low as compared to other industrial branches, the resultant health hazard and possible impact on workers' health has long been, unfortunately, underestimated.

Meanwhile, recent study has indicated, that the majority of nail technicians are concerned about their health status and the effects the occupational exposure to chemicals may impose [2]. In line with this, a growing amount of scientific evidence indicates an increased occurrence of certain adverse health effects possibly associated with exposure to solvents and other volatile organic compounds (VOCs) in occupational setting, including headaches [3,4], skin, eyes and upper airways mucosal irritations [1-3,5], musculoskeletal and reproductive system disorders [1-4,6-8] or bronchial asthma [1,5]. In addition, nail technicians have also been shown to present limited results of pulmonary function tests and increased levels of markers of airways inflammation [6]. Our recent study has revealed that certain adverse effects may be observed also on cellular and molecular levels [9]. Nevertheless, in spite of all this long growing evidence, no occupational standards have been established for this industrial branch so far.

In this study, we investigated the health status of occupationally exposed nail technicians and tried to find out whether this work group is subject to faster health deterioration due to their occupational exposure as compared to office-working non-exposed control subjects. To this end, the subjective assessment of an individual's health status, the prevalence and cumulative incidence of a panel of selected work-related symptoms among nail technicians were compared to those among control subjects. Subsequently, methods of survival analysis were employed to test the intra-group differences in estimated median length of the employment period free of investigated adverse health effects and, finally, we tried to find out whether occupational exposure to VOCs may directly be associated with the incidence of adverse health effects among nail technicians.

\section{MATERIAL AND METHODS Subjects}

In this study, previously described groups of nail technicians and control subjects were used [9]. Briefly, the study group consisted of 145 female nail technicians (aged 2164 years old, median age of 34 years old, interquartile range (IQR): 27-42 years) occupationally exposed to low levels of VOCs recruited from 109 nail and/or beauty salons providing manicure and/or artificial nail sculpturing services in different areas of the city of Łódź in central Poland. All nail technicians declared that they had not been performing any other kind of job previously. Median length of the period of employment among nail technicians was 6 years (IQR: $1-39$ years).

The control group was drawn from a general population restricted to the subjects meeting the main criteria for inclusion in the control group, i.e., a) not being involved in the nail industry, b) spending most of the work time in a sitting position, similar to nail technicians, and c) not being occupationally exposed to VOCs (even in the past). Male subjects were a priori not invited to participate in the control group as all of the nail technicians were females. The control group ultimately counted 152 females aged $19-59$ years 
old (median age of 33 years old, IQR: 27-42 years) and comprised 107 (70\%) general office workers (i.e., clerks, librarians, merchants, secretaries, editors, etc.) and 45 (30\%) academic workers (i.e., senior researchers and lecturers) without any occupational contact with organic solvents. Median length of the period of employment among control subjects was calculated based on their entire employment histories and equaled 7 years (IQR: $0.1-42$ years).

Prior to any further measurements, written and informed consent for participation in this study was obtained from each participant. The study was performed under the guidelines of the Helsinki Declaration for human research and was approved by the Bioethics Committee of the Nofer Institute of Occupational Medicine, Łódź, Poland (resolution No. 5/2011).

\section{Questionnaire survey}

A short researcher-made questionnaire fulfilled by each participant of the study was used for collecting data on the occurrence of adverse health effects subjectively attributed to occupational exposure. The questionnaire was divided into 3 main parts. The first part was aimed at gathering a participant's personal and demographic data, such as her name, surname, age, workplace and the length of the period of employment. In this part, information on current tobacco smoking and alcohol consumption habits of all participants was gathered as well in order to facilitate further adjustment of outcomes of statistical analyses to 2 major confounders - i.e., the current tobacco load (expressed in pack-years (PY), i.e., the number of cigarette packs smoked per day $x$ the number of years lived as a current smoker) and the weekly ethanol ingestion (detailed description is provided elsewhere [9]). The second part of the questionnaire consisted of 3 closed questions and each subject was asked to choose an answer which best describes: 1. Her own subjective assessment of her general health status (possible answers: "excellent," "very good," "good," "fair," "poor").
2. How her health status changed over the last year (possible answers: "much better than year ago," "slightly better than year ago," "unchanged," "slightly worse than year ago," "much worse than year ago").

3. Whether (possible answers: "yes," "no") and for how long she had been suffering from any symptoms causing concerns about her health status, subjectively attributed to working environment.

The third, last part of the questionnaire was aimed at gathering more detailed information on encountered symptoms and each participant was asked to indicate which of the listed symptoms including headache, skin irritation, mucosal irritation, difficult breathing, dyspnea, regular/chronic cough, itchy/runny nose, itchy/watery/reddish eyes, physician-confirmed bronchial asthma, infertility and musculoskeletal disorders (such as shoulder, back or wrist/hand pain) she had been suffering from since the commencement of the job and, if appropriate, to provide information on how long such conditions lasted. In this part, the participants were allowed to mark as many symptoms as appropriate.

For further purposes of this study, the term "adverse health effect" is used to refer to an occurrence of any of the above listed symptoms, as well as an individual assessment of one's own current health status as "good," "fair" or "poor" and reporting the current health status as "slightly worse" or "much worse" as compared to the last year's health status.

\section{Sampling and assessment}

\section{of occupational exposure to VOCs}

Detailed information on air sampling and assessment of occupational exposure of nail technicians to VOCs, performed according to the method by Gjolstad et al. [10] with minor modifications, is provided in the authors' recent study [9]. In this study, occupational exposure to VOCs (including ethanol, acetone, toluene, 2-propanol, 2-butanone, ethyl acetate, isopropyl acetate, n-butyl 
acetate, hexamethyldisiloxane, methyl methacrylate and ethyl methacrylate) was assessed based on the combined exposure to a mixture of VOCs calculated according to the American Conference of Governmental Industrial Hygienists (ACGIH) [11] formula:

$$
\sum \frac{\mathrm{C}}{\mathrm{N}}=\frac{\mathrm{C}_{1}}{\mathrm{~N}_{1}}+\frac{\mathrm{C}_{2}}{\mathrm{~N}_{2}}+\cdots+\frac{\mathrm{C}_{\mathrm{n}}}{\mathrm{N}_{\mathrm{n}}}
$$

where:

$\mathrm{C}_{\mathrm{i}}$ - measured airborne concentration,

$\mathrm{N}_{\mathrm{i}}$ - time-weighted average occupational exposure limit (TWAOEL) for $i$ th component.

\section{Statistical analysis}

All frequency data in the study is provided as both absolute and relative counts. Data collected by means of personal questionnaire was used for inferring both prevalence and cumulative incidence of adverse health effects. The intra-group comparisons of obtained prevalence were performed by means of a generalized linear regression modeling approach assuming the logit model. The strength of associations was assessed by means of the odds ratios (OR) adjusted to between-group differences in participants' age, current tobacco load (expressed in PY) and estimated weekly ethanol ingestion (all 3 considered as confounders), accompanied by corresponding $95 \%$ confidence interval (CI). Raw ORs and risk ratios (RRs) are also provided. Simple intra-group comparisons of prevalence were performed by means of the exact mid-P or the Fisher-Freeman-Halton tests, depending on the number of levels of the analyzed categorical variable. Cumulative incidence of adverse health effects over the period of employment was analyzed employing the methods of survival analysis assuming the Cox proportional hazard model. Cumulative incidence plots, showing the proportion of subjects suffering from adverse health effects against the length of the employment period among both the nail technicians and control subjects were tested for significant intra-group differences by means of the logrank test.

Both groups were characterized by the median length of the period of employment free of given adverse health effect $\left(\mathrm{t}_{50 \%}\right.$ - defined as the length of employment period during which the respective cumulative incidence function reaches the value of 0.5 ) with corresponding 95\% CI (according to Brookmeyer and Crowley [12]) and respective hazard ratio with corresponding 95\% CI. The Cox proportional hazard regression modeling and Kaplan-Meier curves were used for testing the effect of airborne levels of VOCs on the incidence of adverse health effects among nail technicians.

All analyses were performed using R Statistical Software (Foundation for Statistical Computing, Austria) and the Statistica 10 software package (StatSoft, USA). Statistical significance was inferred for $p<0.05$.

\section{RESULTS}

\section{Subjective assessment of health status and the prevalence of symptoms}

The summary of the questionnaire survey of subjective assessment of participants' health status is provided in the Table 1. It may be seen that nail technicians assessed their current health status differently as compared to control subjects, as the distribution of answers is significantly shifted towards the "good," "fair" and "poor" answers in the former ones $(p<0.0001)$. In fact, only $22 \%$ of nail technicians described their current health status as "excellent" or "very good," which was more than 2 times lower as compared to a frequency of almost $45 \%$ for these 2 answers among control subjects (32 out of 145 technicians vs. 68 out of 152 controls: $\mathrm{OR}=0.4,95 \%$ CI: $0.2-0.6$, $\mathrm{RR}=0.5,95 \%$ CI: $0.4-0.7, \mathrm{p}<0.00005)$.

On the other hand, comparing the current health status to that from a year ago, only marginally significant differences in distribution of the answers between 
Table 1. Subjective assessment of the general health status among control subjects and nail technicians occupationally exposed to low levels of volatile organic compounds*

\begin{tabular}{lcc}
\hline \multicolumn{1}{c}{ Health status } & \multicolumn{2}{c}{$\begin{array}{c}\text { Respondents } \\
{[\mathrm{n}(\%)]}\end{array}$} \\
\cline { 2 - 3 } & $\begin{array}{c}\text { nail technicians } \\
(\mathrm{N}=145)\end{array}$ & $\begin{array}{c}\text { control group } \\
(\mathrm{N}=152)\end{array}$ \\
\hline $\begin{array}{l}\text { Subjective assessment of your current health status }{ }^{\mathrm{a}} \\
\text { excellent }\end{array}$ & $2(1.4)$ & $2(1.3)$ \\
very good & $30(20.7)$ & $66(43.4)$ \\
good & $101(69.7)$ & $77(50.7)$ \\
fair & $12(8.3)$ & $6(3.9)$ \\
poor & $0(0.0)$ & $1(0.7)$ \\
Current health status compared to the one from last year & & \\
much better & & $6(3.9)$ \\
slightly better & $12(8.3)$ & $13(8.6)$ \\
unchanged & $11(7.6)$ & $111(76.6)$ \\
slightly worse & $86(59.3)$ & $20(13.1)$ \\
much worse & $31(21.4)$ & $2(1.3)$ \\
\hline
\end{tabular}

* Distribution of answers provided by control subjects and nail technicians to 2 closed questions concerning their current health status. Distributions were tested for significant differences using the exact Fisher-Freeman-Halton test.

${ }^{\mathrm{a}} \mathrm{p}<0.0001$.

${ }^{\mathrm{b}} \mathrm{p}=0.06$.

the 2 groups ( $p=0.06$ ) were found. Nevertheless, thorough inspection of these outcomes may reveal that almost $25 \%$ of nail technicians responded that they felt their current health status was "slightly worse" or "much worse" as compared to the one from the last year, which was significantly higher as compared to $<15 \%$ of controls providing such answers (36 of 145 nail technicians vs. 22 of 152 controls: $\mathrm{OR}=2,95 \% \mathrm{CI}: 1.1-3.5, \mathrm{RR}=1.7$, 95\% CI: 1.1-2.8, p < 0.05).

The prevalence of individual symptoms among nail technicians and control subjects is presented in the Table 2. In general, $61 \%$ of nail technicians confirmed they experienced at least one out of all work-related symptoms considered in the study since the commencement of the job, which was significantly higher as compared to just $17 \%$ of control subjects ( $p$ 0.0001) and associated with increase in both the raw and adjusted odds of symptoms being observable among this working group (raw OR $=7.4$, 95\% CI: 4.3-12.7; adjusted OR $=2.8,95 \%$ CI: 2.1-3.7). Except for physician-confirmed bronchial asthma and infertility, all other symptoms were also significantly more prevalent among nail technicians, with the raw estimate of increased odds ranging from relatively low values observed in the case of eyes-related disorders, such as itchy/ watery/reddish eyes $(\mathrm{OR}=1.8,95 \% \mathrm{CI}: 1.2-2.9, \mathrm{p}<0.05)$ to almost 11 -fold increase in the case of difficulty breathing $(\mathrm{OR}=10.7,95 \% \mathrm{CI}: 2.4-47.1, \mathrm{p}<0.005)$. Following the adjustment to differences in participants' age, current PY and ethanol ingestion, these odds of occurrence of symptoms among nail technicians were reduced considerably but still statistically significant (Table 2). In the case of physician-confirmed bronchial asthma, the intra-group difference in the prevalence was on the edge of statistical significance $(p=0.056)$ with just 0 and 4 such cases among 
Table 2. The prevalence of symptoms subjectively related to working environment among control subjects and nail technicians occupationally exposed to low levels of volatile organic compounds*

\begin{tabular}{|c|c|c|c|c|c|}
\hline \multirow{3}{*}{ Symptom } & \multicolumn{2}{|c|}{$\begin{array}{l}\text { Respondents } \\
{[\mathrm{n}(\%)]}\end{array}$} & \multicolumn{3}{|c|}{ Risk and odds estimates } \\
\hline & \multirow{2}{*}{$\begin{array}{l}\text { nail technicians } \\
\qquad(\mathrm{N}=145)\end{array}$} & \multirow{2}{*}{$\begin{array}{l}\text { control group } \\
(\mathrm{N}=152)\end{array}$} & \multirow{2}{*}{$\begin{array}{l}\text { raw RR } \\
(95 \% \mathrm{CI})\end{array}$} & \multicolumn{2}{|c|}{ OR $(95 \% \mathrm{CI})$} \\
\hline & & & & raw & adjusted \\
\hline Any symptoms in general & $88(60.7)^{\mathrm{a}}$ & $26(17.1)$ & $3.5(2.4-5.1)$ & $7.4(4.3-12.7)$ & $2.8(2.1-3.7)$ \\
\hline Headaches & $36(24.8)^{b}$ & $15(9.9)$ & $2.5(1.4-4.4)$ & $3.0(1.6-5.8)$ & $1.8(1.3-2.5)$ \\
\hline Skin irritation & $27(18.6)^{\mathrm{c}}$ & $6(3.9)$ & $4.7(2.0-11.1)$ & $5.6(2.2-13.9)$ & $2.5(1.5-4.0)$ \\
\hline Mucosae irritation & $52(35.9)^{\mathrm{a}}$ & $13(8.6)$ & $4.2(2.4-7.4)$ & $6.0(3.1-11.6)$ & $2.7(1.9-3.8)$ \\
\hline Difficulty breathing & $18(12.4)^{\mathrm{d}}$ & $2(1.3)$ & $9.5(2.2-40.2)$ & $10.7(2.4-47.1)$ & $3.4(1.6-7.3)$ \\
\hline Dyspnea & $31(21.4)^{\mathrm{e}}$ & $14(9.2)$ & $2.3(1.3-4.2)$ & $2.7(1.4-5.4)$ & $1.6(1.2-2.3)$ \\
\hline Regular/chronic cough & $33(22.8)^{\mathrm{e}}$ & $18(11.8)$ & $1.9(1.1-3.3)$ & $2.2(1.2-4.1)$ & $1.6(1.1-2.2)$ \\
\hline Itchy/runny nose & $102(70.3)^{\mathrm{a}}$ & $71(46.7)$ & $1.5(1.2-1.8)$ & $2.7(1.7-4.4)$ & $1.7(1.3-2.2)$ \\
\hline Itchy/watery/reddish eyes & $84(57.9)^{\mathrm{f}}$ & $66(43.4)$ & $1.3(1.1-1.7)$ & $1.8(1.2-2.9)$ & $1.4(1.1-1.7)$ \\
\hline Physician-confirmed bronchial asthma & $4(2.8)^{g}$ & $0(0.0)$ & - & - & - \\
\hline Infertility & $3(2.1)$ & $1(0.7)$ & $3.1(0.3-29.9)$ & $3.2(0.3-31.0)$ & $1.8(0.6-5.8)$ \\
\hline Musculoskeletal disorders & $13(9.0)^{\mathrm{f}}$ & $3(2.0)$ & $4.5(1.3-15.6)$ & $4.9(1.4-17.5)$ & $1.9(1.0-3.7)$ \\
\hline
\end{tabular}

* The absolute (relative) counts of control subjects and nail technicians reporting individual symptoms which they subjectively attribute to working environment (i.e., occurred after the job commencement and vanished during off-work days) have been provided. Raw odds ratios (ORs) and risk ratios (RRs) together with respective $95 \%$ confidence intervals (CIs) were calculated based on respective contingency tables. The ORs adjusted to subjects' age, current-tobacco load and weekly ethanol ingestion (by means of alcoholic beverages) as well as the provided levels of significance were inferred using the generalized nonlinear regression modeling approach assuming the logit model.

${ }^{\mathrm{a}} \mathrm{p}<0.0001 ;{ }^{\mathrm{b}} \mathrm{p}<0.001 ;{ }^{\mathrm{c}} \mathrm{p}<0.0005 ;{ }^{\mathrm{d}} \mathrm{p}<0.005 ;{ }^{\mathrm{e}} \mathrm{p}<0.01 ;{ }^{\mathrm{f}} \mathrm{p}<0.05 ;{ }^{\mathrm{g}} \mathrm{p}=0.056$ (exact Fisher's test).

the controls and nail technicians, respectively. No statistically significant differences in the prevalence of infertility between the 2 groups analyzed were found in the study.

\section{Length of the employment period}

\section{free of adverse health effects}

With increasing length of employment period, the cumulative proportion of subjects assessing their current health status in worse ratings (i.e., "good," "fair" or "poor") and the proportion of subjects reporting that their current health status had been "slightly worse" or "much worse" than last year were both found to rise significantly faster among nail technicians as compared to control subjects (Figures 1a and $1 b)$. This is consistent with significantly shorter $t_{50 \%}$ and significantly increased hazard of the occurrence of these 2 adverse health effects in the group of nail technicians as compared to controls (Table 3 ).

The outcomes for symptoms reported by subjects are very similar. The cumulative incidence of any concern-causing symptoms combined together was again found to rise with increasing period of employment in both analyzed groups, nevertheless it showed up to rise significantly faster among nail technicians as compared to controls (Figure 1c). Consequently, the estimated $t_{50 \%}$ for the occurrence of all concern-causing symptoms combined together was significantly shorter among nail technicians as compared to controls (12 years vs. 33 years, $p<0.0001$ ), consistent with almost 4-times increased hazard of the occurrence of any concern-causing symptoms among the technicians (Table 3). 

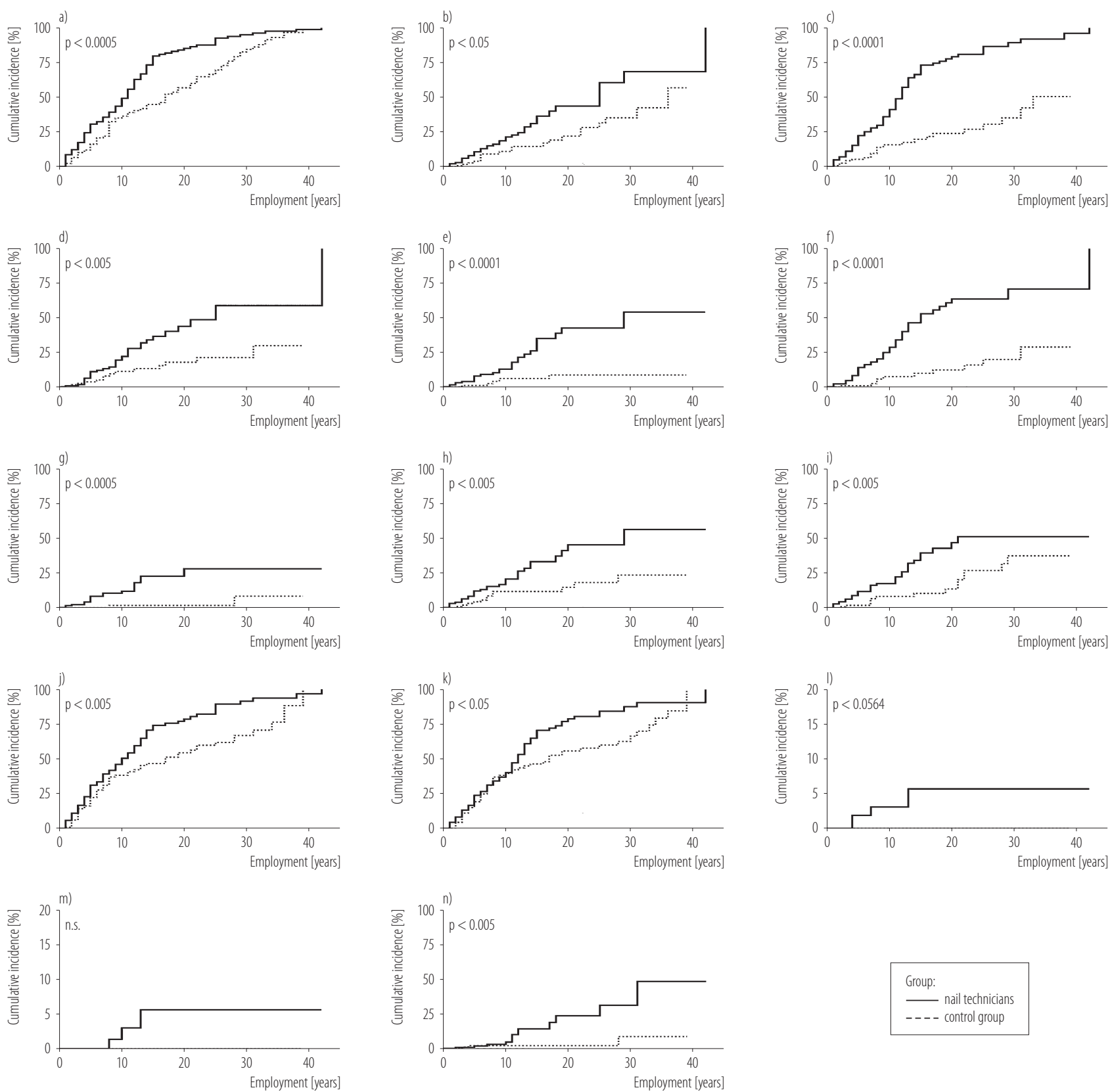

n.s. - not statistically significant.

The cumulative proportion of subjects in respective groups classifying their current health status as: a) "good," "fair" or "poor," b) "slightly worse" or "much worse" compared to the last year's health status.

Subsequent panels show the cumulative incidence of: c) all symptoms combined together, d) headaches, e) skin irritations, f) mucosal irritations, g) difficulty breathing, h) dyspnea, i) regular/chronic cough, j) itchy/runny nose, k) itchy/watery/reddish eyes, l) physician-confirmed bronchial asthma, $\mathrm{m}$ ) infertility, n) musculoskeletal disorders. Inter-group differences between cumulative incidence curves were tested for significance by means of log-rank test.

Summary outcomes of survival analyses are provided in Table 3.

Fig. 1. Cumulative incidence plots for adverse health effects investigated in the study among nail technicians occupationally exposed to low levels of volatile organic compounds and control group 
Table 3. Summary of survival analyses regarding control subjects and nail technicians occupationally exposed to low levels of volatile organic compounds*

\begin{tabular}{|c|c|c|c|c|}
\hline \multirow[b]{2}{*}{ Adverse health effect } & \multicolumn{2}{|c|}{$\mathrm{t}_{50 \%}(\mathrm{IQR})$} & \multirow[b]{2}{*}{$\mathrm{HR}(95 \% \mathrm{CI})$} & \multirow[b]{2}{*}{$\mathrm{p}$} \\
\hline & $\begin{array}{l}\text { nail technicians } \\
\quad(\mathrm{N}=145)\end{array}$ & $\begin{array}{c}\text { control group } \\
(\mathrm{N}=152)\end{array}$ & & \\
\hline \multicolumn{5}{|l|}{ Assessment of current health status } \\
\hline “good”, "fair” or “poor” & $10(9-12)$ & $17(11-22)$ & $1.7(1.3-2.3)$ & $<0.0005$ \\
\hline "slightly worse" or "much worse" than 1 year ago & $25(17-42)$ & $36(26-$ n.a. $)$ & $1.9(1.1-3.2)$ & $<0.0500$ \\
\hline \multicolumn{5}{|l|}{ Symptoms } \\
\hline Any symptoms in general & $12(10-13)$ & 33 (28-n.a.) & $3.9(2.7-5.7)$ & $<0.0001$ \\
\hline Headaches & 25 (17-n.a.) & $>39$ (n.a.-n.a.) & $2.6(1.5-4.4)$ & $<0.0050$ \\
\hline Skin irritation & 29 (18-n.a.) & $>39$ (n.a.-n.a.) & $5.6(2.8-11.2)$ & $<0.0001$ \\
\hline Mucosae irritation & $15(12-20)$ & $>39$ (n.a.-n.a.) & $4.7(2.9-7.7)$ & $<0.0001$ \\
\hline Difficulty breathing & - & - & $9.0(3.8-21.7)$ & $<0.0005$ \\
\hline Dyspnea & 29 (18-n.a.) & $>39$ (n.a.-n.a.) & $2.7(1.5-4.8)$ & $<0.0050$ \\
\hline Regular/chronic cough & 21 (15-n.a.) & $>39$ (29-n.a.) & $2.6(1.5-4.6)$ & $<0.0050$ \\
\hline Itchy/runny nose & $10(8-12)$ & $17(11-25)$ & $1.6(1.2-2.2)$ & $<0.0050$ \\
\hline Itchy/watery/reddish eyes & $12(10-13)$ & $17(10-28)$ & $1.4(1.0-2.0)$ & $<0.0500$ \\
\hline Physician-confirmed bronchial asthma & - & - & - & 0.0564 \\
\hline Infertility & - & - & $2.8(0.4-19.6)$ & n.s. \\
\hline Musculoskeletal disorders & - & - & $5.1(1.9-13.5)$ & $<0.0050$ \\
\hline
\end{tabular}

* For both groups involved in the study. The median length of the period of employment free of respective adverse health effect $\left(\mathrm{t}_{50 \%}\right)$ and the respective hazard ratio (HR) have been provided, both with corresponding $95 \%$ confidence intervals (CIs). In the cases where the $t_{50 \%}$ was available at least for nail technicians, the respective value of $\mathrm{t}_{50 \%}$ for the controls was indicated as exceeding the maximum length of the period of employment in the study for that group (> 39 years). If the values of $\mathrm{t}_{50 \%}$ were not available for any of the 2 groups, only dash (-) is provided. The levels of significance were inferred using the log-rang test.

IQR - interquartile range.

n.a. - not available (indicated when the limit of the interquartile range (IQR) was not available).

n.s. - not statistically significant.

When headaches, skin irritations, mucosal irritations, difficult breathing, dyspnea, regular/chronic cough, itchy/ runny nose, itchy/water/reddish eyes and musculoskeletal disorders were considered separately, the obtained cumulative incidence curves were also found to differ significantly between the 2 groups of subjects suggesting faster increase of the proportion of subjects suffering from respective symptoms and increased hazard of the occurrence of these symptoms among nail technicians as compared to controls (Table 3 ). For the majority of these symptoms (including headaches, skin irritations, mucosal irritations, difficult breathing, dyspnea, regular/ chronic cough and musculoskeletal disorders), direct comparison of $t_{50 \%}$ was impossible due to the fact that they were relatively rare among control subjects but the cumulative incidence curves presented in the Figure 1 and data in the Table 3 clearly indicate these values to be significantly lower among nail technicians as compared to control subjects, irrespectively of which an individual symptom is considered. 
Concerning the physician-confirmed asthma, limited occurrence of asthma in both analyzed groups did not allow the respective $t_{50 \%}$ to be calculated, yet the respective cumulative incidence curves differed marginally between the 2 groups indicating a marginally increased hazard of asthma among nail technicians $(\mathrm{p}=0.0564)$ (Table 3). The cumulative incidence curves for infertility were not found to differ significantly between the 2 groups suggesting no differences in the hazard of its occurrence between the groups.

\section{Effect of exposure to VOCs on the occurrence}

\section{of adverse health effects among nail technicians}

According to data provided in our recent study [9], the occupational exposure of nail technicians to a mixture of VOCs according to the ACGIH formula was low, with median level of 0.033 (IQR: 0.014-0.081) and maximum level not exceeding the value of 0.333 . Employing the Cox proportional hazard regression modeling, the level of such combined exposure was found to significantly affect the rate in which the proportion of nail technicians suffering from work-related symptoms rises as the length of the employment period increases but only when all symptoms were combined together $(\mathrm{p}=0.05)$.

The Figure 2 shows the Kaplan-Meier curves obtained for nail technicians exposed to the highest (33\% of the threshold limit) and lowest ( $0.2 \%$ of the threshold limit) observed combined exposure to VOCs. The higher rate of reduction of the proportion of symptom-free subjects among nail technicians exposed to higher levels of the mixture of VOCs was consistent with almost 5-times increased hazard of the occurrence of any symptoms among them as compared to those exposed to lower levels (hazard ratio $(\mathrm{HR})=4.9,95 \% \mathrm{CI}: 1-24.1)$. Combined exposure to a mixture of airborne VOCs was, however, not found to significantly modulate the hazard of occurrence of any symptoms, when symptoms considered in this study were analyzed separately (data not shown).

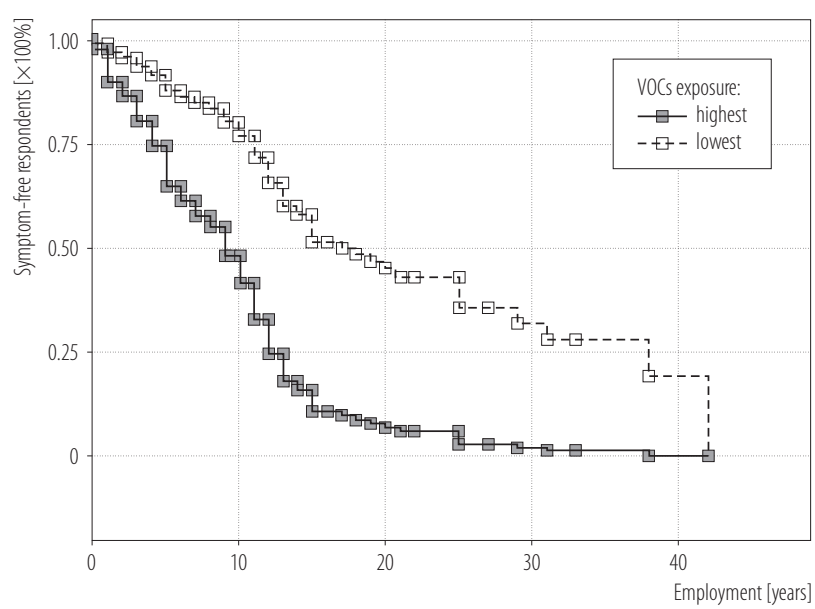

The above chart presents the Kaplan-Meier curves showing the proportion of nail technicians with the highest (33\% of the threshold limit) and lowest ( $0.2 \%$ of the threshold limit) VOCs exposure observed in the study not suffering from any of the symptoms analyzed in the study. Squares indicate the censored data. The difference between the curves was characterized by hazard ratio (HR) and tested for significance by means of the log-rank test.

$\mathrm{HR}=4.9,95 \%$ CI: $1-24.1, \mathrm{p}=0.05$.

Fig. 2. Effect of combined exposure to a mixture of volatile organic compounds (VOCs) on the occurrence of all symptoms combined together among nail technicians

\section{DISCUSSION}

The hereby presented results show that occupationally exposed nail technicians comprise a work group with significantly increased prevalence of certain health symptoms, including headache, skin, nose and eyes mucosal irritation, respiratory, musculoskeletal disorders, and asthma as compared to controls. Prevalence of such symptoms among nail technicians has already been investigated by several previous studies [1-8] and although the respective frequencies of individual symptoms among nail technicians vary from study to study, all of them seem to be consistent in suggesting that these symptoms are more likely to occur among nail technicians.

In line with this, information on an individual assessment of current health status gathered in this study indicates that nail technicians more frequently assess their individual health status in worse categories as compared to 
controls, either when it comes to assessing their current health status or comparing it to the one from the last year. Worse health status and the fact that adverse health effects among nail technicians often get better when away from work may plausibly explain the previously reported increased frequency of concerns about the health effects of chemicals used at work [1,2].

Symptoms analyzed in this study may be divided into 3 main classes. The first class involved the mucous membranes-related symptoms, such as respiratory or eyesrelated problems. In context of these symptoms, it is worth noticing that respiratory problems associated with unusually high occupational exposures to VOCs have been reported to occur among professional painters or furniture workers quite long ago $[13,14]$. Later cross-sectional studies have shown that respiratory problems and adverse allergic airways symptoms are likely to occur also in the case of "ordinary" exposure to environmental (i.e., mostly indoor) VOCs, not exceeding any limits set for occupational environment $[15,16]$. A question has thus arisen as what might be the possible mechanism underlying such adverse health effects among subjects exposed to VOCs irrespectively of the exposure level.

A recent South Korean study has revealed that both the environmental exposure to VOCs as well as respiratory problems and reduced pulmonary functions observed among exposed subjects are directly associated to levels of oxidative stress markers and oxidative damage of mucous membranes in small airways. This indicates that oxidative stress may play a crucial role in induction of respiratory problems caused by VOCs [17]. In a series of in vitro and animal-model studies, oxidative damage of airways was found to be caused by reactive oxygen species generated as a result of toxic action of VOCs and was shown to lead to upregulation of a number of inflammation mediators released from eosinophils, neutrophils, alveolar macrophages and epithelial cells promoting inflammatory response of the cells. Moreover, reaction oxygen species act as second messengers in intracellular cascades and are thus capable of modulating many intracellular processes, including inflammation and immune responses [18].

Volatile organic compounds, on the other hand, have been shown to directly interfere with dendritic cell functions, which is supposed to further modify the cellular immune response and may lead to allergic inflammation [19-24]. Since both the short-term exposure to high concentrations of VOCs as well as long-term exposure to lower (indoorrelevant) ones were used in hereby cited studies, it may be assumed that inflammation response in airways of subjects exposed to VOCs is triggered in the case of both environmental and occupational exposure, with the latter one being associated with allergic airways inflammation, as well. Considering the specific aspects of nail technicians' occupational exposure to VOCs, particularly the close proximity of the sources of VOCs facilitating the contact of VOCs with their eyes and airways, and the rather prolonged exposure to these substances, both airways inflammation and allergy may be assumed to develop in this workgroup, manifesting themselves in the form of the respiratory problems analyzed in this study. As it is well known that except for mucous membranes of airways, tissues responding to toxic action of VOCs include also the mucous membranes of eyes and the skin on face, neck and hands, it is possible to assume that symptoms related to eyes and skin reported by nail technicians may be underlain by similar mechanisms based on generation of oxidative stress.

The second major class of adverse health effects analyzed in this study comprised musculoskeletal disorders, also often reported by nail technicians. These disorders comprise a certainly different class of problems. These disorders were consistently found to be of increased prevalence among nail technicians occupationally exposed to low levels of VOCs in almost all previous studies [1-4,6-8] and our study clearly stands in line with all of them. It has been proposed that such disorders might be associated with the necessity of holding compromised postures in order 
to be able to access the field of treatment, what is probably associated with increased muscular effort and fatigue. In the case of visually demanding and precise operations within a relatively fixed field of treatment, the muscular strain of the neck, back, hands, wrists and shoulders was proposed to be even further magnified, which may result in increased prevalence of this kind of symptoms in the work group analyzed in this study as compared to other officebased environments in which the workers spend most of their times sitting [7,25].

Finally, infertility was investigated in this study as the last adverse health effect. Our study failed to provide enough evidence in favor of significantly increased risk thereof in this work group, which may be, at least partly, attributed to a limited study size resulting in just 4 incidences of infertility in total. Unfortunately, we did not gather any information on other reproductive disorders such as spontaneous abortions or menstrual cycle disorders, as all of these (including infertility) were reported previously to occur among hairdressers which were exposed to VOCs much similar to those used in nail industry during their work time [26-29]. Moreover, it was found that the risk of such disorders is associated with the daily amount of work time and the level of chemical exposure [28] and that it was reduced following the enactment of bans on the use of selected chemicals supposed to cause reproductive toxicity [29]. Such increased risk of reproductive disorders has not been, however, found among females performing both hairdressing and nails sculpturing services (referred to as "cosmetologists") [30-33]. This inconsistency may be to some extent explained by the fact, that nail treatments are usually associated with handling lower amounts of chemicals and thus the resultant exposure is substantially lower as compared to that in the case of hairdressers [34].

Moreover, it has to be emphasized, that the current knowledge on the effect of occupational exposure to mixtures of VOCs such as those found in nail salons has been relatively limited so far and not much is known on modulatory effects of possible bias factors (such as work-related mental stress) [8,34]. Modest occupational reproductive effects of exposure to low-level VOCs have, however, been reported to occur also in other working environments with exposure to low-level VOCs, such as among women working in the liquid crystal display manufacture [35] but the exposure level reported by this study has been much higher as compared to those reported in our study (even though still referred to as the "low-level exposure").

As suggested earlier, VOCs are assumed to affect female menstrual functions mainly by means of hormonal disruption, which is supposed to be plausible mechanism of reproductive disorders [35]. Either way, no increased risk of infertility among nail technicians has been seen in our study.

Despite the fact that all hereby discussed effects of occupational exposure on health status of nail technicians have already been suggested by several above cited studies, the vast majority of them has been designed as simple cross-sectional studies, providing mere endpoint estimates of the raw frequencies of work-related symptoms at a given time-point. The new point of view provided by our study is based on survival analysis techniques employed in order to perform quantitative assessment of the hazard of the occurrence of above discussed adverse health effects and estimate the expected median length of the employment period until their occurrence among nail technicians and compare it with control subjects. Based on hereby presented data, it is obvious that as the length of employment period increases, the number of nail technicians suffering from work-related adverse health effects rises significantly faster as compared to control subjects, a phenomenon which we observed in the case of all analyzed adverse health effects except for physician-diagnosed bronchial asthma (for which only marginally significant differences were found) and infertility. In other words, the period of employment free of above discussed adverse health effects $\left(\mathrm{t}_{50 \%}\right)$ was found to be significantly shorter among nail 
technicians as compared to controls, which is consistent with increased hazard of such health effects among nail technicians.

Changes in cumulative incidence of work-related adverse health-effect among subjects occupationally exposed to low-level VOCs in the function of the length of employment period clearly corroborate the suggestion provided by a study in which the length of the employment period has been considered in relation to occupational exposure of nail technicians, the only one which we have been able to find to date [6]. This study has revealed that the length of employment period and intriguingly also the time of contact with methacrylates (key chemical substances in nail industry) were both positively correlated with the extent of airways inflammation and significant worsening of pulmonary functions among nail technicians. This may, at least to some extent, provide some explanation why the cumulative incidence of respiratory symptoms increases faster among nail technicians as compared to unexposed control subjects.

It is of note, that except for itchy/runny nose, itchy/watery/ reddish eyes, dyspnea and skeleton disorders, in the case of which the cumulative incidence curves obtained for the 2 groups enrolled in the study begin to separate from each other after some 10 years of employment, respective curves obtained for all the other symptoms analyzed in the study diverge from each other as soon as just right after the job commencement.

It indicates that the onset of individual symptoms reported by nail technicians is not simultaneous and that certain factors exist which cause some of them to occur later. However, the nature of these factor remains unknown at this stage of research and this might probably be at least partially caused by our limited knowledge on how the mixtures of VOCs affects one's health. Such lack of knowledge is perhaps reflected also in our inability to satisfactorily interpret the outcomes of the Cox regression modeling performed in this study, where significant effects were observed only if all symptoms were combined together. It is however worth emphasizing that in our study, the group of nail technicians was compared to control subjects at the same age, which is crucial due to known discrepancies in health status between the young working population and a general one [36]. Thus, the comparison presented in our study might be interpreted as free of any bias possibly introduced by referencing the outcomes in nail technicians to those of a general population.

Several limitations need to be, however, taken into account while interpreting the outcomes of this study. First, one has to keep in mind that although some of the symptoms investigated in this study (such as difficulty breathing, dyspnea, regular/chronic cough, itchy/runny nose or bronchial asthma) may be considered as directly affected by pathological processes ongoing in airways and although outcomes of survival analyses and the Cox regression modeling seem to indicate the importance of the length of the employment period and the exposure to VOCs, any conclusions concerning the direct causality between these symptoms and occupational exposure-mediated pathologies in airways of exposed subjects must be drawn carefully, as no clinical examinations were performed in our study. Besides that, only static and short-term breathingzone area measurements were performed in order to assess the level of occupational exposure of nail technicians, which could have been thus slightly underestimated and may have led to compromised outcomes of the Cox proportional hazard regression modeling. Also, a bias may have been introduced into the study due to the fact that it is quite plausible that salons which do not meet certain criteria of occupational hygiene refused to participate in the study.

\section{CONCLUSIONS}

In this study we provide evidence on increased prevalence of adverse health effects among nail technicians occupationally exposed to low levels of VOCs as compared 
to unexposed office-based control subjects. In line with this, nail technicians subjectively assess their health status in worse ratings as compared to controls. The survival analysis has revealed a significantly increased hazard of almost all symptoms investigated in this study (except for physician-confirmed bronchial asthma and infertility), consistent with significantly shortened period of employment free of these symptoms. Although the outcomes of regression modeling remain ambiguous, higher levels of combined exposure to a mixture of VOCs were found to increase hazard of the occurrence of symptoms investigated in this study almost five-fold. All these outcomes combined together indicate that nail technicians are subject to faster health deterioration, which may be assumed to be caused by occupational exposure to low levels of VOCs, even though the levels of such exposure do not exceed any occupational limits.

\section{REFERENCES}

1. Roelofs C, Azaroff LS, Holcroft C, Nguyen H, Doan T. Results from a community-based occupational health survey of Vietnamese-American nail salon workers. J Immigr Minor Health. 2008;10(4):353-61, https://doi.org/10.1007/s10903007-9084-4.

2. Quach T, Nguyen K-D, Doan-Billings P-A, Okahara L, Fan C, Reynolds P. A preliminary survey of Vietnamese nail salon workers in Alameda County, California. J Community Health. 2008;33(5):336-43, https://doi.org/10.1007/ s10900-008-9107-7.

3. Park S-A, Gwak S, Choi S. Assessment of occupational symptoms and chemical exposures for nail salon technicians in Daegu city, Korea. J Prev Med Public Health. 2014;47(3): 169-76, https://doi.org/10.3961/jpmph.2014.47.3.169.

4. Quach T, Gunier R, Tran A, von Behren J, Doan-Billings P-A, Nguyen K-D, et al. Characterizing workplace exposures in Vietnamese women working in California nail salons. Am J Public Health. 2011;101(S1):S271-6, https://doi.org/10.21 05/AJPH.2010.300099.
5. Lazarov A. Sensitization to acrylates is a common adverse reaction to artificial fingernails. J Eur Acad Dermatol Venereol. 2007;21(2):169-74, https://doi.org/10.1111/j.14683083.2006.01883.x.

6. Reutman SR, Rohs AM, Clark JC, Johnson BC, Sammons DL, Toennis CA, et al. A pilot respiratory health assessment of nail technicians: Symptoms, lung function, and airway inflammation. Am J Ind Med. 2009;52(11):868-75, https://doi.org/10.1002/ajim.20751.

7. Harris-Roberts J, Bowen J, Sumner J, Stocks-Greaves M, Bradshaw L, Fishwick D, et al. Work-related symptoms in nail salon technicians. Occup Med (Lond). 2011;61(5): 335-40, https://doi.org/10.1093/occmed/kqr096.

8. Pak VM, Powers M, Liu J. Occupational chemical exposures among cosmetologists: Risk of reproductive disorders. Workplace Health Saf. 2013;61(12):522-8, https://doi. org/10.3928/21650799-20131121-01.

9. Grešner P, Stępnik M, Król MB, Świercz R, PieniążekSmok A, Twardowska E, et al. Dysregulation of markers of oxidative stress and DNA damage among nail technicians despite low exposure to volatile organic compounds. Scand J Work Environ Health. 2015;41(6):579-93, https://doi.org/ 10.5271/sjweh.3523.

10. Gjolstad M, Thorud S, Molander P. Occupational exposure to airborne solvents during nail sculpturing. J Environ Monit. 2006;8(5):537-42, https://doi.org/10.1039/B601917J.

11. American Conference of Governmental Industrial Hygienists. Transactions of the Twenty-Fifth Annual Meeting of the American Conference of Governmental Industrial Hygienists; 1963 May 6-10; Cincinnati (OH), USA.

12. Brookmeyer R, Crowley J. A confidence interval for the median survival time. Biometrics. 1982;38(1):29-41, https://doi. org/10.2307/2530286.

13. Weislander G, Nordback D, Edling C. Airway symptoms among house painters in relation to exposure to volatile organic compounds (VOCS) - A longitudinal study. Ann Occup Hyg. 1997;41(2):155-66, https://doi.org/10.1093/ annhyg/41.2.155. 
14. Talini D, Monteverdi A, Benvenuti A, Petrozzino M, di Pede F, Lemmi M, et al. Asthma-like symptoms, atopy, and bronchial responsiveness in furniture workers. Occup Environ Med. 1998;55(11):786-91, https://doi.org/10.1136/ oem.55.11.786.

15. Elliott L, Longnecker MP, Kissling GE, London SJ. Volatile organic compounds and pulmonary function in the Third National Health and Nutrition Examination Survey, 19881994. Environ Health Perspect. 2006;114(8):1210-4, https:// doi.org/10.1289/ehp.9019.

16. Nielsen GD, Larsen ST, Olsen O, Løvik M, Poulsen LK, Glue C, et al. Do indoor chemicals promote development of airway allergy? Indoor Air. 2007;17(3):236-55, https://doi. org/10.1111/j.1600-0668.2006.00468.x.

17. Yoon HI, Hong Y-C, Cho S-H, Kim H, Kim YH, Sohn JR, et al. Exposure to volatile organic compounds and loss of pulmonary function in the elderly. Eur Respir J. 2010;36(6): 1270-6, https://doi.org/10.1183/09031936.00153509.

18. Valko M, Rhodes CJ, Moncol J, Izakovic M, Mazur M. Free radicals, metals and antioxidants in oxidative stress-induced cancer. Chem Biol Interact. 2006;160(1):1-40, https://doi. org/10.1016/j.cbi.2005.12.009.

19. Wang F, Li C, Liu W, Jin Y. Effect of exposure to volatile organic compounds (VOCs) on airway inflammatory response in mice. J Toxicol Sci. 2012;37(4):739-48, https://doi.org/ 10.2131/jts.37.739.

20. Wang F, Li C, Liu W, Jin Y, Guo L. Effects of subchronic exposure to low-dose volatile organic compounds on lung inflammation in mice. Environ Toxicol. 2014;29(9):1089-97, https://doi.org/10.1002/tox.21844.

21. Bönisch U, Böhme A, Kohajda T, Mögel I, Schütze N, von Bergen $\mathrm{M}$, et al. Volatile organic compounds enhance allergic airway inflammation in an experimental mouse model. PLoS One. 2012;7(7):e39817, https://doi.org/10.1371/journal.pone.0039817.

22. Fischäder G, Röder-Stolinski C, Wichmann G, Nieber K, Lehmann I. Release of MCP-1 and IL-8 from lung epithelial cells exposed to volatile organic compounds. Toxicol In
Vitro. 2008;22(2):359-66, https://doi.org/10.1016/j.tiv.2007. 09.015 .

23. Feltens R, Mögel I, Röder-Stolinski C, Simon J-C, Herberth G, Lehmann I. Chlorobenzene induces oxidative stress in human lung epithelial cells in vitro. Toxicol Appl Pharmacol.2010;242(1):100-8,https://doi.org/10.1016/j.taap. 2009.09.020.

24. Mörbt N, Tomm J, Feltens R, Mögel I, Kalkhof S, Murugesan K, et al. Chlorinated benzenes cause concomitantly oxidative stress and induction of apoptotic markers in lung epithelial cells (A549) at nonacute toxic concentrations. J Proteome Res. 2011;10(2):363-78, https://doi.org/10.1021/pr1005718.

25. Kilbom A. Repetitive work of the upper extremity: Part I Guidelines for the practitioner. Int J Ind Ergon. 1994;14: 51-7, https://doi.org/10.1016/0169-8141(94)90005-1.

26. Ronda E, García AM, Sánchez-Paya J, Moen BE. Menstrual disorders and subfertility in Spanish hairdressers. Eur J Obstet Gynecol Reprod Biol. 2009;147(1):61-4, https://doi. org/10.1016/j.ejogrb.2009.07.020.

27. Baste V, Moen BE, Riise T, Hollund BE, Øyen N. Infertility and spontaneous abortion among female hairdressers: The Hordaland Health Study. J Occup Environ Med. 2008;50(12):1371-7, https://doi.org/10.1097/JOM.0b013e318 1858723.

28. John EM, Savitz DA, Shy CM. Spontaneous abortions among cosmetologists. Epidemiology. 1994;5(2):147-55, https://doi.org/10.1097/00001648-199403000-00004.

29. Kersemaekers WM, Roeleveld N, Zielhuis GA. Reproductive disorders due to chemical exposure among hairdressers. Scand J Work Environ Health. 1995;21(5):325-34, https:// doi.org/10.5271/sjweh.46.

30. Peretz J, Gallicchio L, Miller S, Greene T, Zacur H, Flaws JA. Infertility among cosmetologists. Reprod Toxicol. 2009;28(3): 359-64, https://doi.org/10.1016/j.reprotox.2009.05.068.

31. Peters C, Harling M, Dulon M, Schablon A, Torres Costa J, Nienhaus A. Fertility disorders and pregnancy complications in hairdressers - A systematic review. J Occup Med Toxicol. 2010;5:24, https://doi.org/10.1186/1745-6673-5-24. 
32. Gallicchio L, Miller S, Greene T, Zacur H, Flaws JA. Cosmetologists and reproductive outcomes. Obstet Gynecol. 2009;113(5):1018-26,https://doi.org/10.1097/AOG.0b013e31 81a1f906.

33. Gallicchio L, Miller SR, Greene T, Zacur H, Flaws JA. Health outcomes of children born to cosmetologists compared to children of women in other occupations. Reprod Toxicol. 2010;29(3):361-5, https://doi.org/10.1016/j.reprotox. 2010.01.011.

34. Halliday-Bell JA, Gissler M, Jaakkola JJK. Work as a hairdresser and cosmetologist and adverse pregnancy outcomes. OccupMed(Lond).2009;59(3):180-4,https://doi.org/10.1093/ occmed/kqp017.
35. Lin C-C, Huang C-N, Wang J-D, Hwang Y-H, Shie R-H, Chang Y-Y, et al. Exposure to multiple low-level chemicals in relation to reproductive hormones in premenopausal women involved in liquid crystal display manufacture. Int J Environ Res Public Health. 2013;10(4):1406-17, https:// doi.org/10.3390/ijerph10041406.

36. McDonald C, Checkoway H. Chapter 18. Study design. In: McDonald C, editor. Epidemiology of work related diseases. London: BMJ Books; 2000. p. 385. https://doi.org/ 10.1002/9780470695005.ch18.

This work is available in Open Access model and licensed under a Creative Commons Attribution-NonCommercial 3.0 Poland License - http://creativecommons.org/ licenses/by-nc/3.0/pl/deed.en. 